\title{
AC 2010-25: PROMOTING EFFECTIVE COMMUNICATION IN GLOBAL ENGINEERING PROJECTS
}

\section{Joanna DeFranco, Pennsylvania State University}

Joanna F. DeFranco is an Engineering faculty member at Penn State University. She earned her

B.S. in Electrical Engineering and Math from Penn State, M.S. in Computer Engineering from Villanova, and earned her Ph.D. in Computer and Information science from New Jersey Institute of Technology. Previous to entering academia, Dr. DeFranco held a number of positions in industry and government, including software engineer for Motorola in Horsham, PA and an Electronics Engineer for the Naval Air Development Center in Warminster, PA. She has published a number of articles in journals and conference proceedings in the area of collaborative problem solving, group cognition, global engineering, and computer forensics.

\section{Fadi Deek, New Jersey Institute of Technology}

Fadi P. Deek received his B.S. Computer Science, 1985; M.S. Computer Science, 1986; and Ph.D. Computer and Information Science, 1997 all from New Jersey Institute of Technology (NJIT). He is Dean of the College of Science and Liberal Arts, Professor of Information Systems, Information Technology, and Mathematical Sciences at NJIT where he began his academic career as Teaching Assistant in 1985. He is also a member of the Graduate Faculty - Rutgers University Business School. Dr. Deek maintains an active funded-research program. His research interests include Learning/Collaborative Systems, Software Engineering, Open Source Development, Computer Science Education.

\section{Raghvinder Sangwan, Pennsylvania State University, Great Valley}

Raghvinder S. Sangwan, an Associate Professor of Software Engineering at the Pennsylvania State University's School of Graduate Professional Studies, holds a Ph.D. in Computer and Information Sciences from Temple University. He joined Penn State in 2003 after a 7+ year career in industry, where he worked mostly with large software-intensive systems in the domains of healthcare, automation, transportation and mining. His teaching and research involves analysis, design, and development of software systems, their architecture, and automatic and semi-automatic approaches to assessment of their design and code quality. Dr. Sangwan has several peer reviewed publications in these areas. He actively consults for Siemens Corporate Research in Princeton, NJ and also holds a visiting scientist appointment at the Software Engineering Institute at the Carnegie Mellon University in Pittsburgh, PA. He is also a senior member of the Institute of Electrical and Electronics engineers (IEEE)and the Association of Computing Machinery (ACM). 


\title{
Promoting Effective Communication in Global Engineering Projects
}

\begin{abstract}
Effective communication plays a key role in the success of engineering teams. However, achieving a high level of communication when developing projects globally can be challenging. An organization's learning capacity, its familiarity with the cultural diversity of its team members, and its information technology support for project planning, data management, group communication and collaboration among geographically distributed teams, are some key factors that can help overcome this challenge.
\end{abstract}

\section{Introduction}

Through a study conducted in 2008 by NASA, communication was identified as one of the five top level themes their highly valued Systems Engineers possess ${ }^{21}$. The purpose of the NASA study is to accelerate the development of critical Systems Engineering behaviors and to ensure these behaviors were developed for the next generation of highly regarded System Engineers. In reality, effective communication is extremely important in any type of engineering. For example, in requirements engineering effective communication is imperative during processes that support knowledge acquisition and understanding of stakeholder needs ${ }^{13}$.

The increased need to improve communication efforts is evident due to many engineering disciplines increasing their development efforts across different sites in different geographic locations and across different time zones. Major factors that have contributed to this globalization trend include cheaper labor, the need for specialized skills and expertise, and corporate mergers. For example, the outsourcing of various functions or activities of the software business has become increasingly prevalent leading to "capability sourcing" particularly when companies' expertise falls short in some technical areas. In addition, some companies already have offices that span the world creating a globally distributed technical asset, although they may still outsource software development for strategic reasons.

Globalization can, however, bring on big challenges. It is well known, for instance, that effective communication plays a key role in the performance of product development teams ${ }^{2,3,4,5}$. Researchers have shown that well-coordinated teams demonstrate a higher level of overall performance, especially when their tasks are interrelated and compactly situated ${ }^{6,7}$. Achieving the desired levels of coordination among geographically distributed teams can be extremely challenging, however, due to the negative impact that increased distance has on communication ${ }^{8}$. Research shows that a mere 100 meters of separation results in a significant drop in communication between team personnel ${ }^{2}$. The communication degradation may increase with distance; however, beyond 100 meters it becomes almost irrelevant whether collaborators are 
located in two different buildings, cities, countries, or continents: communication is degraded and team performance suffers serious setbacks in all these contexts.

These negative effects are even more pronounced when the teams are located several time zones apart with minimal overlap in working hours ${ }^{5}$. Under these conditions, collaborators must rely heavily on asynchronous interactions (i.e., different individuals providing input to the task at different times); if the work is also not closely related, and the teams are not communicating well, problems can escalate quickly.

This paper explores some of the challenges and possible factors that could contribute to successful communication during globalized engineering efforts. We present the results of a survey instrument that showed key factors that can help overcome some challenges in communicating effectively in engineering teams.

\section{Related Work}

When developing complex systems there typically are many people involved. Many of these project stakeholders are likely to be not at the same physical location. Critical communication needs to take place among them in order for a quality product to result. In the next few sections we explore possible factors that may affect communication efforts.

\section{Cultural Differences}

Cultural challenges are significant factors in the success of global software development ${ }^{10}$. Difficulties can arise in communicating one's point of view and ideas clearly and precisely across sites because of differences in culture, language, and values ${ }^{17}$. Additionally, differences in time zone forces most communication to be asynchronous. As a result, it is not only that much less communication may take place; it is also a less effective form of communication ${ }^{1}$. A lot of time is wasted coordinating and clarifying understandings via e-mail, voice mail, and message boards. These issues also affect other important aspects of the project such as lack of information about who is an expert in what, and who is responsible for which aspect of the project ${ }^{12}$.

Education is certainly one possible remedy for these potential conflicts. Educating team members about the differences in work culture and values could have a positive effect on communication. An experiment with students participating in a globalized project where half of the students were located in the US and the other half were in India yielded interesting results ${ }^{14}$. Students had a positive experience when regularly injecting the cultural differences into classroom discussions. For example, speakers who had experience in both the Indian and American workplace were invited to the classroom to highlight cultural differences and similarities. The students also experienced a week of socialization prior to the experiment, which contributed to the success of the study. Furthermore, the students communicated with one another about expectations and other viable communication methods. 
The communication overhead of a project where there are no cultural differences can be challenging. Adding the cultural difference factors increases this overhead significantly. It is suggested that to bridge the cultural gap a cultural liaison could be established ${ }^{18}$. The liaison's role would be established at the outset and would be played by a project manager, or an architect at the remote site.

\section{Managerial Factors}

Globalization and outsourcing require modified managerial skills. Many executives remain unprepared for the transformation that comes with globalization ${ }^{1}$. The needed skills, such as communication, project management, business analysis and team management, are much more complex in a virtual setting ${ }^{14}$. A study was carried out among software developers and found that their communication is much more effective when there are management efforts to encourage communication between development teams ${ }^{16}$. This would include established protocols of meetings between team members as well as keeping team members informed of major decisions regarding projects. This same study surveyed developers and found that communication was more effective when management made efforts to improve organizational activities and processes such as designating resources to assist employees in case of technical difficulties and also participated in the definition of the project development planning. Another significant outcome showed an increase in effective communication from management efforts to involve users during the project development. The project, it appears, has a higher probability of success if the user is informed of the progress and able to evaluate progress during the development.

A collaborative effort when developing complex systems, whether the team members are in the same physical location or not, also requires IT support. IT support includes applications to assist in project management, data management and collaboration. Project management is necessary to keep track of deadlines, data management to keep track of source code, and communication to keep track of team memory, effective idea exchange and reaching consensus. All of these resources need management buy-in to be implemented and to be successful.

\section{Learning and Group Cognition}

Team members involved in development projects can be quite diverse in their approaches to problem solving which obviously can cause conflict. When working on a specific problem (Problem A), the management will most likely be faced with an additional problem (Problem B) which deals with how to effectively manage the diversity among the problem solvers ${ }^{11}$. In order to be productive, a team must spend more time working on problem A and less on problem B. One way for management to accomplish more of a focus on problem $A$, the real engineering problem to be solved, is to understand not only how to manage knowledge diversity but also to understand the process of group cognition.

Knowledge diversity can be managed by offering training and promoting continuous learning in an organization. Another way to manage knowledge differences is to actively promote discussion of team goals, strategies, and work practices among team members. For example, in a 
systems engineering project the strategies/goals/practices include developing systems requirements, specifications, and architectures.

The thought processes developers use is also fundamental to understanding engineering design and development ${ }^{18}$. Since the cognitive activities that occur in a group are even more varied and more complex than those in individuals ${ }^{19}$, it is still more essential to understand the role of such processes in group problem solving. Their complexity is compounded because one is faced not merely with individual cognitive activities, but with the interplay of cognitive activities among individuals.

With any type of engineering development, one particularly interesting cognitive effect is cognitive bias. Cognitive bias refers to the tendency of individuals to be consistent and predictable in their behavior with respect to the kind of errors they make. Such biases operate at both the individual and the group level. Various techniques have been proposed to reduce cognitive biases including obvious improvements such as using empirical as opposed to intuitive analysis, as well as less obvious strategies such as systematically seeking what is called disconfirmatory information, or systematically evaluating the tradeoffs for each project guideline $^{18}$. For example, intuitive approaches, which refer to immediate cognition not reinforced by an explicit process, leave greater room for error than method-based approaches. Stacy \& Macmillian (2005) describe seeking disconfirmatory information as asking negatively phrased empirical questions such as "How will I know if the feature does not work?" or "How will I know if this is not the cause of the problem?" Finally, it is suggested that when applying project guidelines which are defined as one engineers opinion based on a past experience, the engineers needs to evaluate each of these guidelines as a two-sided tradeoff. For example, one engineer may believe that Java is the best language for web development. It's important to communicate to the team the advantages and disadvantages of using Java for this particular project. These techniques reduce cognitive bias by moderating the cognitive impact of previous experiences, which cognition tends to bring to mind first, even though the previous experience may be irrelevant or invalid in the current situation.

Group cognition is a coordinated, distributed cognitive process, the objective of which is to create a shared, distributed understanding of a problem at a team level ${ }^{20}$. Though the importance of this area of research is increasing, information technologies are frequently not designed with the requirements of such teams in mind and they suffer accordingly. Nosek calls the cognitive actions and interchanges that occur during collaborative problem solving group sensemaking. Nosek's model identifies three conditions required to create this kind of knowledge in a problem solving group: distributed knowledge, distributed cognition, and coordinated cognitive processing among group members. Proper coordination of cognitive processing allows the members of a group to have comparable knowledge of a problem area ${ }^{15}$. All of this has a strong relationship to effective communication and can manage some of the diversity among the problem solvers, thus, reducing problem B. 


\section{Research Hypotheses}

Effective communication, as pointed out previously, is an essential success factor when collaborating. With globalized engineering development, however, it has been observed there is much less communication and also less effective communication ${ }^{12}$.

Hypotheses, shown in Table 1, are designed to determine the role of organizational background and IT infrastructure in producing effective communication when collaboratively designing and developing engineering products among geographically distributed teams. Organizational background refers specifically to two factors:

1. Learning capacity: referring to the promotion of learning about the project and knowledge needed to achieve the project goals. The team members in order to realize full learning capacity should also be motivated to learn and to cooperate.

2. Cultural familiarity: This refers to the level of familiarity of the culture of all team members as well as the level of comfort team members experience when interacting and communicating with team members.

IT infrastructure is also made up of the following two factors:

1. IT system support for organizational and management standards and policies such as project planning and data management.

2. IT system support for group communication and collaboration.

Effective communication is measured by four factors: informed scale, task clarity, communication effectiveness (among all stake holders), and coordination success. The informed scale is defined as how well the engineers felt they and the project managers were informed on the project status ${ }^{6}$. Task clarity is measuring how well the engineer understands their tasks and responsibilities. Reducing task uncertainty decreases the amount information that needs to be processed to make decisions ${ }^{9}$. Communication effectiveness measures generally the level of how the engineers perceived the participation, satisfaction and understanding among the technical staff, development teams, the organization and the users of the system. And finally the coordination success can be implied from effective communication ${ }^{10}$.

The results from the survey instrument (described in more detail in the next section) are used to determine if organizational background and IT infrastructure influence effective communication.

Table 1. Effective Communication Hypotheses

H1: A higher level of organizational background information will result in a higher level of effective communication. Supported

H1A: Effective communication of an engineering team is higher for teams with a higher level of organizational learning capacity. Supported 
H1B: Effective communication of an engineering team is higher when they have a higher level of cultural familiarity amongst their team members. Supported

H2: A higher level of IT support will result in a higher level of effective communication. Supported

H2A: Effective communication of an engineering team is higher for the team with higher levels of IT that provides support to organizational and management standards and policies. Supported

H2B: Effective communication of an engineering team is higher for the team with higher levels of IT group communication support. Supported

\section{Methodology and Results}

The 43 question online survey was adopted from an earlier study by Mohtashami (2006) and modified for our purposes. The survey was divided into three sections. The first section asked the participants how well the organization engages in learning and asked them to evaluate the level of cultural differences among their team members. The next section asked questions to evaluate the information technology support that is provided to the team. Information technology support is referring to everything from project management applications to group communication software. And finally, the last section evaluated the communication effectiveness explained in detail in the previous section. The survey was taken by 59 professional graduate engineering students at the Penn State University. The students answering the survey averaged 8.2 years experience working collaborative projects. The results were extremely positive as indicated by the supported hypotheses in the previous section of this paper.

Figure 1 shows a fitted line plot resulting from an analysis of variance analysis (ANOVA). The data was a summary of both the questions pertaining to how well the people in the organization can engage in learning with their team members as well as the understanding of cultural differences between collaborating team members. The ANOVA showed a $p$ value of 0.0 (less than .1\%) significance. Both of these factors have had an increasing effect on the communication effectiveness among a team.

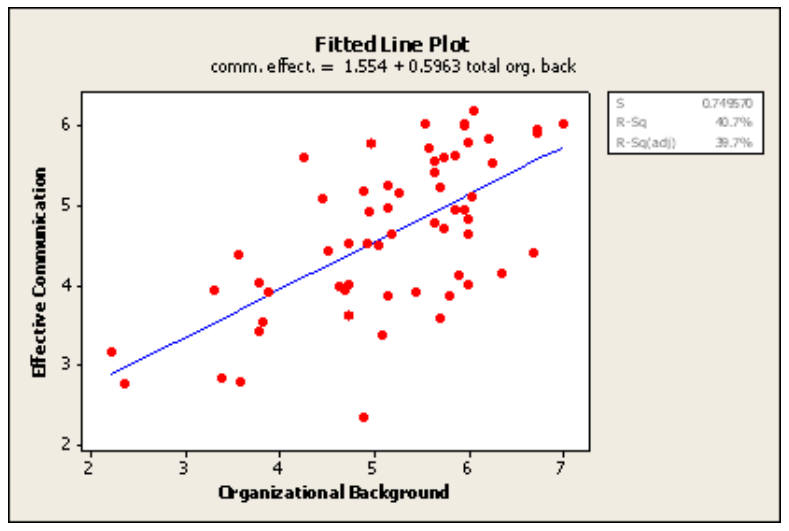

Figure 1. Effective communication versus organizational background 
Figure 2 shows a fitted line plot resulting from another ANOVA analysis. The data used in this plot summarizes both of the questions pertaining to the level of support that IT systems provides to organizational management standards and policies and the level of support that IT system provides for group communication and collaboration. The ANOVA showed a $\mathrm{p}$ value of 0.0 (less than .1\%) significance. These results indicate that the technology support also had an increasing effect on the communication effectiveness of the team.

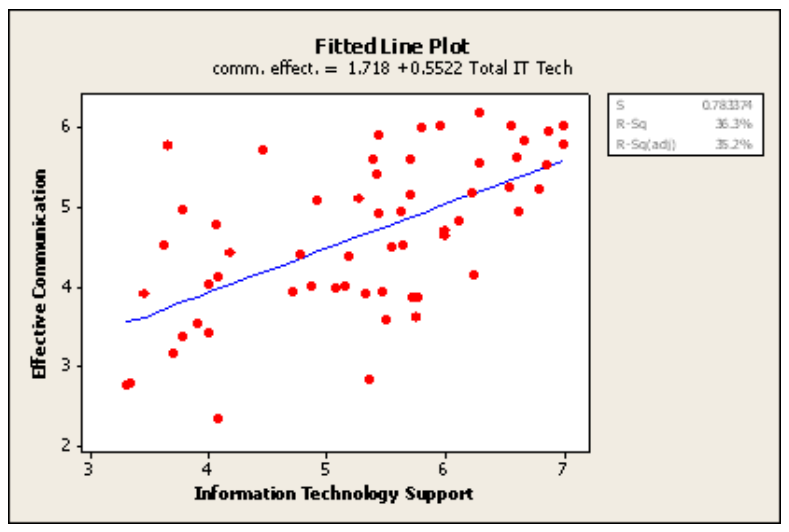

Figure 2. Effective communication versus information technology support

\section{Conclusions}

The success of collaboration in engineering design and development teams is derived from the domain skills of the discipline as well as social and cognitive psychology and supporting technology. All are fundamental to understanding how collaborative development groups interact and produce their products. As a result, globalization affects engineering design and development both in direct and indirect ways. Direct effects are those that are relevant to every stage of the development process including processes and standards. Indirect effects are those related to collaboration, the appropriate interaction environment and group organization. Business considerations and economic benefits have to accept factors beyond the sales, marketing and managerial realm to include cultural, linguistic, and geographic considerations.

Our contention is that team members who understand the cultural differences between team members also will have effective communication within their team. Our results showed that a team shifting some effort towards the indirect effects such as understanding a team member's culture, language and general work practices can increase communication effectiveness. In addition, a small shift in focusing on learning by discussing team goals, strategies, and training to accomplish various parts of the project can also make a significant impact on communication effectiveness. 


\section{Bibliography}

1. Gottfredson, M., Puryear, R., Phillips, S., "Strategic Sourcing: From Periphery to the Core", Harvard Business Review, February 2005.

2. Allen, T., Managing the flow of technology: Technology transfer and the dissemination of technological information within the R\&D organization, MIT Press, Cambridge, MA, 1984.

3. Allen, T., Lee, D., and Tushman, M., "R\&D performance as a function of internal communication, project management, and the nature of the work," IEEE Transactions on Engineering Management, vol. 27, 1984, pp. $2-12$.

4. McDonough, E., Kahn, K., and Griffin, A.,"Managing communication in global product development teams," IEEE Transactions on Engineering Management, vol. 46, 1999, pp. 375-386.

5. Sosa, M., Eppinger, S., Pich, M., McKendrick, D., and Stout, S., "Factors that influence technical communication in distributed product development: an empirical study in the telecommunications industry," IEEE Transactions on Engineering Management, vol. 49, no. 1, February, 2002, pp. 45-58.

6. Kraut, R., and Streeter, L., "Coordination in software development," Communications of the ACM, vol. 38, no. 3, 1995, pp. 69-81.

7. Malone, T. and Crowston, K. "The interdisciplinary study of coordination," ACM Computing Surveys, vol. 26, no. 1,1994 , pp. 87-119.

8. Carmel, E., and Agarwal, R., "Tactical approaches for alleviating distance in global software development," IEEE Software, vol. 18, no. 2., 2001.

9. Galbraith, J.R. Organization Design, Reading, MA: Addison Wesley, 1977.

10. Espinosa, J. A., Kraut, R. E., Slaughter, S. A., Lerch, J. F., Herbsleb, J. D.; Mockus, A., "Shared Mental models, Familiarity and Coordination: A multi-method study of distributed software teams", Twenty-third International Conference of Information systems, 2002, pp 425-433.

11. Kirton, M., Adaption-Innovation: In the context of Diversity and Change, Routledge 2006.

12. Herbsleb, J., "Global Software Engineering: The Future of Socio-technical Coordination", Future of Software Engineering, 2007.

13. Damian, D., "Stakeholders in Global Requirements Engineering: Lessons Learned from Practice", IEEE Software, March/April 2007.

14. Adya, M., Nath, D., Sridhar, V., "Bringing Global Sourcing into the Classroom: Experiential Learning via Software Development Project", SIGMIS - CPR, April, 2007.

15. DeFranco-Tommarello, J., Collaborative Problem Solving and Program Development Model, Ph.D., Dissertation, New Jersey Institute of Technology, Newark, NJ, 2002.

16. Mohtashami, M., The Antecedents and Impacts of Information Processing Effectiveness in Inter-Organizational Collaborative Software Development, Ph.D., Dissertation, Rutgers University, NJ, 2006.

17. Mockus, A., Hersleb, J., "Challenges of Global Software Developmnet", International Software Metrics Symposium (METRICS), 2001.

18. Stacy, W., Macmillian, J., "Cognitive Bias in Software Engineering", Communications of the ACM, Volume 39, Number 6, June 1995, pp. 57-63.

19. Hohmann, L., Journey of the Software Professional, Prentice Hall PTR, New Jersey, 1997.

20. Nosek, J., "The Case for Collaborative Programming", Communications of the ACM, Volume 41, Number 3, 1998, pp. 105-108.

21. Williams, C., Derro, M., "NASA Systems Engineering Behavior Study", NASA Office of the Chief Engineer, October 2008. 


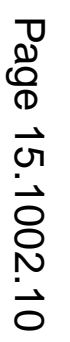

